\title{
TIME RESOLVED ENERGY MEASUREMENT OF THE TESLA TEST FACILITY BEAM THROUGH THE ANALYSIS OF OPTICAL TRANSITION RADIATION ANGULAR DISTRIBUTION
}

\author{
M. Castellano, A. Cianchi, V. Verzilov INFN-LNF CP 1300044 Frascati (Italy) \\ L. Catani, G. Orlandi INFN-Roma 2
}

\begin{abstract}
The study of the energy stability along the macropulse of the TESLA Test Facility Linac (TTFL) [1] was obtained by the measurement of the angular distribution of the Optical Transition Radiation (OTR). This technique does not require a dispersive section and can be performed at any point of the beam line.

Measurements have been performed with different settings of the RF low level control and at different values of the beam current. An energy variation along the macropulse was observed in a good agreement with the measured energy spread of the whole macrobunch.

The analysis of the OTR angular distribution pattern allows also, to some extent, to evaluate the beam angular spread.
\end{abstract}

\section{INTRODUCTION}

The energy stability along the macropulse of the TESLA Test Facility Linac (TTFL) [1] is the result of an accurate timing between the RF pulse and the beam injection to compensate for the beam loading. A digital feedback and feedforward algorithm takes care of fast and slow fluctuations. A measurement of the energy stability is required for a fine tuning of the algorithm parameters.

Typically, energy and energy spread measurements are performed in the dispersive section at the end of the transport line, where a dipole magnet bends the beam by $20^{\circ}$ and drives it to the dump.

Energy variation along the macropulse can be measured by means of the strip-line beam position monitor available in the same dispersive section, but the large beam width, of the same order or larger than the linear range of the monitor, strongly reduces the accuracy of the measure.

Instead, we used the angular properties of the Optical Transition Radiation (OTR) emitted by the beam crossing a thin aluminum foil, to realize a time resolved energy measure. This measure does not require a dispersive section and can be done, in principle, at every section of the accelerator.

A sensor is placed in the focal plane of a thin achromatic lens to allow the imaging of the OTR angular distribution.

The radiation is emitted in a cone of semiaperture $1 / \gamma$, $\gamma$ being the relativistic factor of the incident particles.

In our case the sensor is the cathode of an intensified CCD camera that has several advantages with respect to a normal CCD. It provides the possibility of a fast controlled gate (down to $200 \mathrm{~ns}$ ), allows a 12 bits dynamics and has a high signal to noise ratio.
We integrated the signal over 1 microsecond. Delaying the gate by steps of 1 microsecond, we could follow the whole macrobunch evolution. To ensure the bunch to bunch stability we repeated the measure several times.

\section{RESULTS}

We performed two different measures at different stages of the commissioning of TTF.

The first one was realized with the so-called injector I delivering a beam current of $6 \mathrm{~mA}$ at a repetition rate of $216 \mathrm{MHz}$, with a single accelerating module in operation.

A $45^{\circ}$ beam splitter was used to perform in the same time the measure of both the beam energy (with the ICCD) and the spot size (with a normal CCD). The reflectivity of the beam splitter depends on the radiation polarization, resulting in a different intensity in the horizontal and vertical planes (see Fig.1.)

We used a thin achromatic lens of $\mathrm{f}=200 \mathrm{~mm}$ focal length. Peak positions, and hence the beam energy, were found by applying a fit to the profile obtained from the OTR image along the vertical line crossing the center.

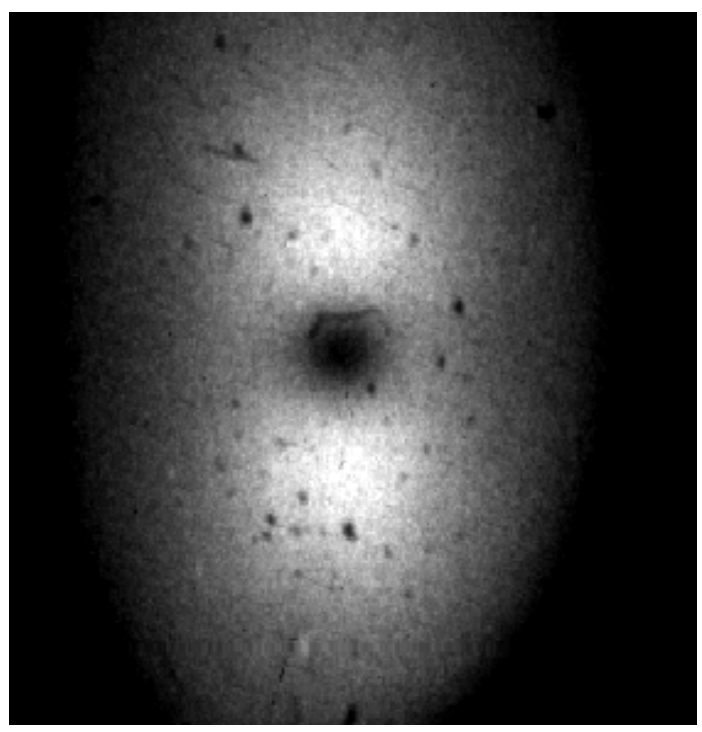

Fig 1 An image of the OTR angular distribution. The difference between horizontal and vertical planes is due to the use of a beam splitter

Fig.2 displays the results of the measurement for two different beam energies. 


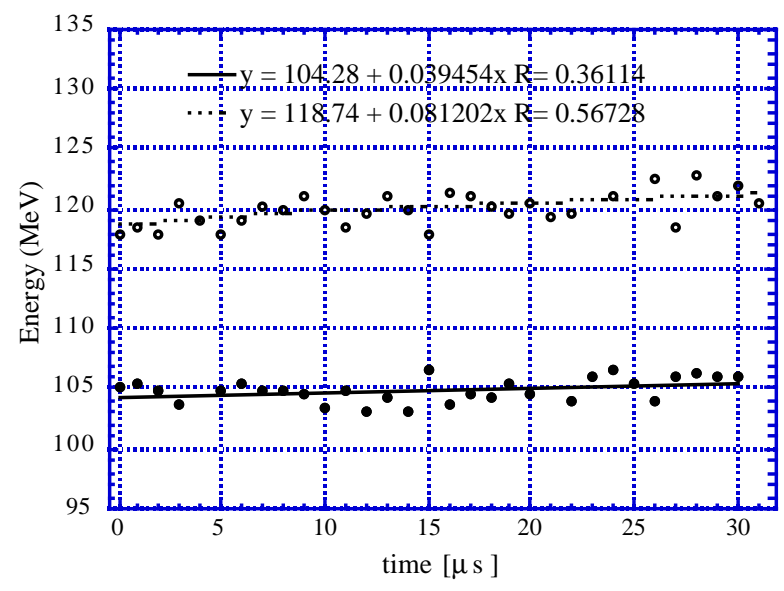

Fig 2 - Variation of the beam energy along the macropulse for two different RF gradients.

In the case of the higher energy, that is the usual working point, the total energy variation along the macropulse is of the order of $2.5 \%$. This result is in a good agreement with the measure made in the dispersive section, which gives a rms energy spread of about $1 \%$ over the whole macrobunch.

At a second stage of the commissioning, we operated with the injector II, which supplies a different pulse time structure. The repetition frequency is $1 \mathrm{MHz}$, so that the integration over 1 microsecond allows the detection of a single micropulse. For these measurements we used a beam current of $1 \mathrm{~mA}$, equivalent to $1 \mathrm{nC}$ of charge for each micropulse, as required for the FEL operation [2].

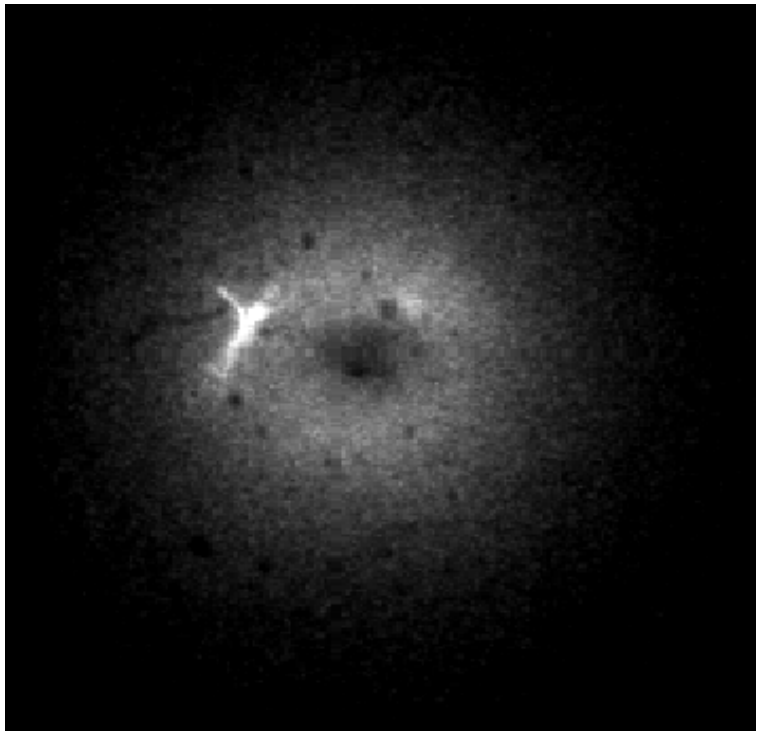

Fig 3 - A typical image of the OTR angular distribution with the injector II

In addition, the installation of a second accelerating module increased the energy of about a factor of 2. In Fig. 3 a typical image recorded in this condition is shown.

We performed measurements for three different values of the loop gain in the low-level RF feedback control system for the accelerating modules. This is an important parameter for the control of the gradient during the RF flat top and, thus, of the energy stability along the macropulse. Fig.4 shows the results for the loop gain equal to 44,36 and 27 , respectively.
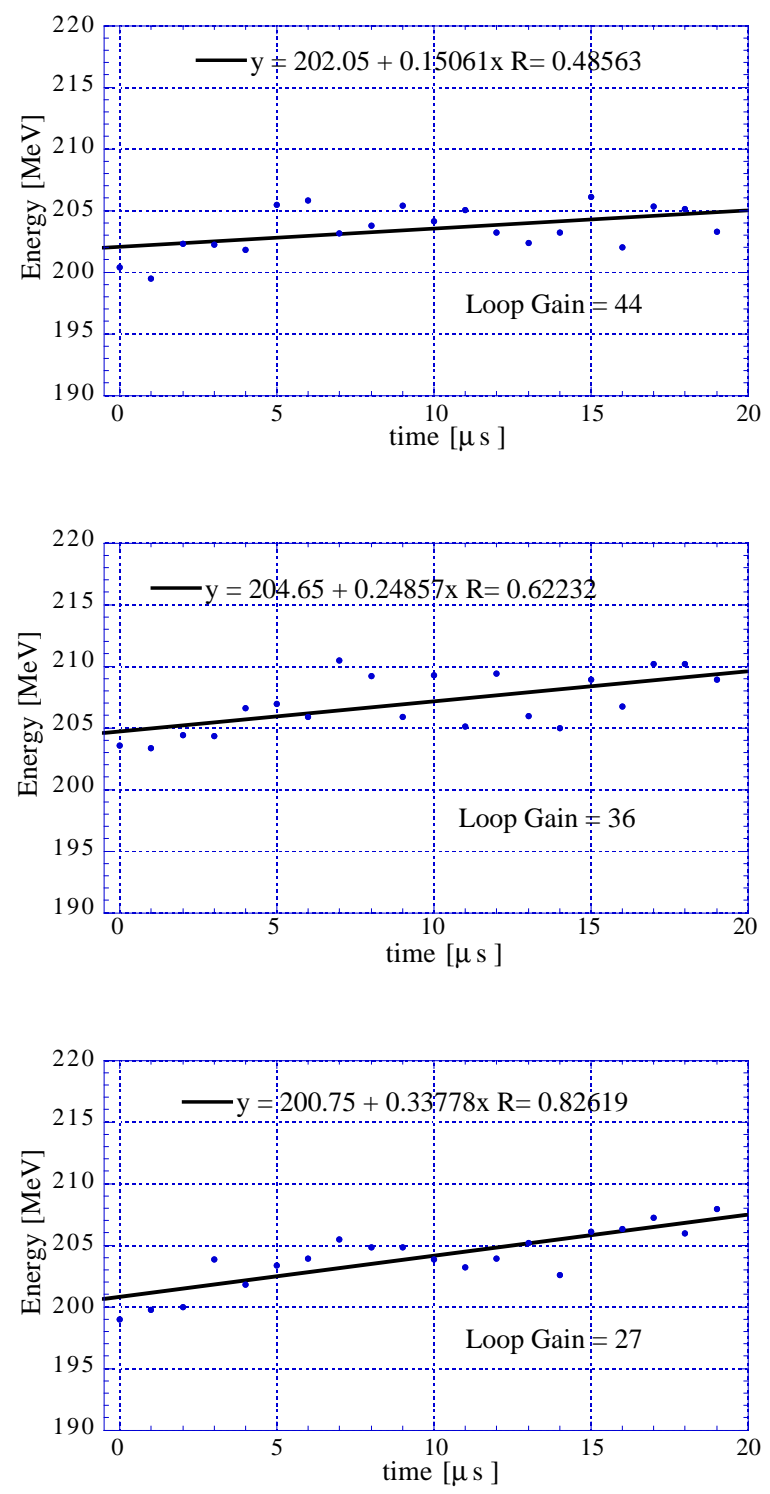

Fig. 4 - Energy variation in the macropulse with three different setting of the RF loop gain

Although the statistical fluctuation of the points is rather large, it is possible to conclude that the energy stability is better at larger values of the loop gain.

A more accurate and systematic work is required in order to determine the best setting for the low-level RF control, and it will be performed in the next run this Summer.

As a by-product of our measurements, we found that an information about the beam angular spread could be obtained by the analysis of the OTR angular distribution profiles.

In Figs. 5 and 6 an example of the vertical profile is presented. 


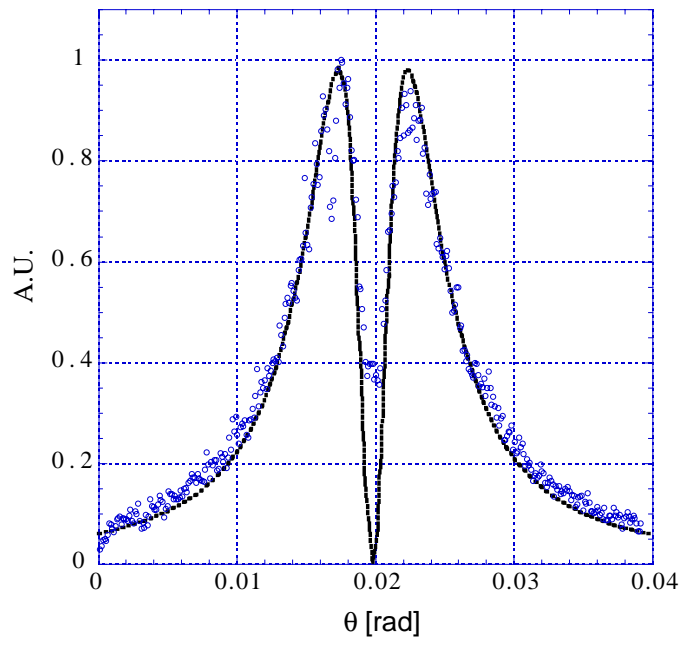

Fig 5 - A profile for the vertical plane of the OTR angular distribution and fitting curve for a parallel beam

The solid line in Fig. 5 is a fit according to the theoretical prediction for a zero divergence beam.

The fit in Fig 6 takes into account the beam divergence and reveals a better agreement with the experimental data.

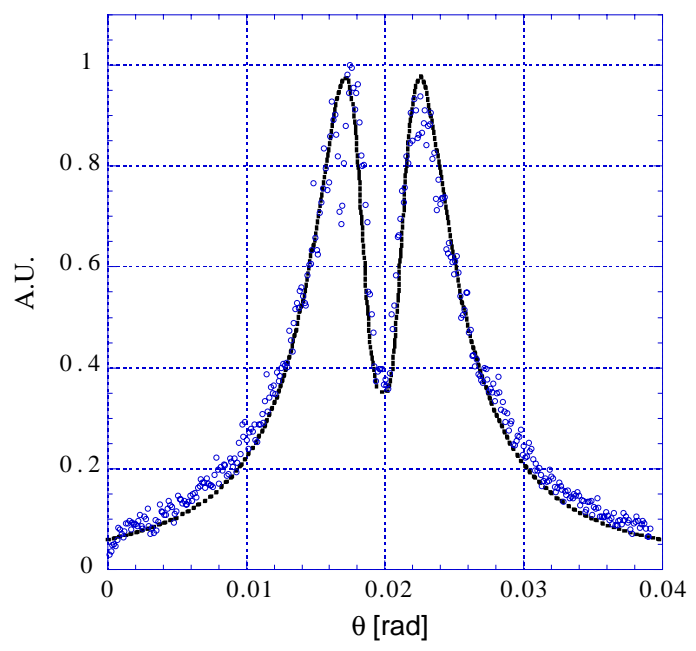

Fig. 6 - Profile of the OTR angular distribution with a fitting function taking into account the beam angular divergence.

A rms angular spread of $0.7 \mathrm{mrad}$ is obtained in this case. Unfortunately, for this data set, in order to avoid the distortion introduced by the beam splitter, we were not able to measure simultaneously the beam spot size. Nevertheless, a spot size value of about $200 \mu \mathrm{m}$ could be obtained, for the same conditions, from the emittance measurements performed just before. According to this value, a normalized emittance of about $60 \mathrm{~mm} \mathrm{mrad}$ can be estimated. This is fully consistent with the measured values that ranged from 50 to $100 \mathrm{~mm} \mathrm{mrad}$, depending on beam transport conditions.

\section{CONCLUSION}

We used the properties of the OTR to monitor the energy variation along the macropulse of TTF beam.

Two different measures were performed at different stages of the commissioning, and in both cases we found an increase of the energy along the macropulse.

We have proven that the loop gain value of the lowlevel RF control can affect the time energy dependence, and that the correct value for this parameter must still be found. A more accurate measurement is needed to explore better this point.

A preliminary analysis shows that it is possible to obtain from the same data also the beam angular divergence, that, together with the measure of the spot size, can give an independent emittance measurement.

To improve the measure accuracy, we plan to use a lens with a longer focal length. A higher number of images for each setting point is also needed in order to reduce the statistical fluctuations.

\section{REFERENCES}

[1] D. Edwards (editor), TESLA Test Facility Linac Design Report, DESY TESLA 95-01 (1995)

[2] A VUV Free Electron Laser at the TESLA Test Facility at Desy - Conceptual Design Report DESY TESLA-FEL 95-03 (1995) 